\title{
Robust Fault Detection using Polytope-based Set-membership Consistency Test
}

\author{
Joaquim Blesa, Vicenç Puig and Jordi Saludes
}

\begin{abstract}
This article proposes a robust set-membership fault detection method based on the use of polytopes to bound the parameter uncertainty set. The proposed polytope-based fault detection algorithm is able to handle systems with bounded parameter variation between samples. It is shown that consistency checks indicating faults can be performed in a natural manner with a polytope description of the feasible parameter set.. Finally, an application example is given, which demonstrates how the algorithm works on a simulated process (a four-tank system). A comparison with a zonotope-based fault detection approach recently proposed in the literature is included.
\end{abstract}

\section{INTRODUCTION}

$\mathrm{T}$ HE principle of model-based fault detection is to test whether the measured system inputs and outputs are consistent with the system behaviour described by a faultless model. If the measurements are inconsistent with the model of the faultless system, the existence of a fault is proved. The residual vector usually describes the consistency check between the predicted and the real behaviour. Ideally, the residuals should only be affected by the faults. However, the presence of disturbances, noise and modelling errors causes the residuals to become nonzero and thus interferes with the detection of faults. Therefore, the fault detection procedure must be robust against these undesired effects [1]. In case that parametric uncertainties are taken into account, the healthy system model should include a vector of uncertain parameters bounded by sets that contains all possible parameter values when the system operates normally.

The set-membership approach is a passive robust fault detection approach that is based on computing explicitly the set of parameters or states that are consistent with the measurements. When a measurement is found to be inconsistent with this set, a fault is assumed to have occurred. As an exact representation of the set of parameters consistent with the measurements is hard to calculate, outer bounds are often used instead, using algorithms coming from

Manuscript received May 28, 2010. This work was supported in part by the grant CICYT HYFA DPI2008-01996 and WATMAN DPI2009-13744 of Spanish Ministry of Education.

J. Blesa and V. Puig are with Automatic Control Department (ESAII), Technical University of Catalonia (UPC), Pau Gargallo 5, 08028 Barcelona, Spain. (e-mail: \{ joaquim.blesa,vicenc.puig\}@upc.edu)

J. Saludes is with Applied Mathematics Department (MA2), Technical University of Catalonia (UPC) Carrer Colom 11, 08222 Terrassa, Spain (email:jordi.saludes@upc.edu). set-membership identification [2]. This is the approach adopted in this article.

Set-membership fault detection algorithms have been the subject of a number of publications. They can be classified according to how the approximation of the feasible set of parameters or states is represented or parameterized. For example in [3] the feasible parameter set was approximated with an ellipsoid. In [4], a numerically robust ellipsoid state estimation algorithm was presented. In [5], a fault detection scheme based on orthotopic sets was presented. In [6], a consistency state-estimation-based fault detection scheme was presented that uses the recursive optimal bounding parallelotope. In [7], a robust polytope state observation algorithm was proposed. In [8], a fault detection method that approximates the feasible parameter by outer zonotopes was proposed in such a way that is able to handle systems with invariant parameters, with parameter variation bounded between samples.

The main contribution of this paper is to provide a robust fault detection method that takes into account bounded variation in parameters and focus on their application to passive robust fault detection as in [8] but using polytopes to bound the parameter uncertainty. On the other hand, the calibration of the algorithm is also considered.

The structure of the paper is the following: Section II is dedicated to the problem definition. In Section III, the fault detection procedure is presented for the invariant case and for the varying case. In Section $I V$, the varying fault detection test implemented with polytopes is explained. In Section $V$, practical issues are addressed such how to tune the fault detection algorithm. In Section VI, a case study based on a well known control benchmark (the four-tank system) is used to show the performance of the robust fault detection method presented in this paper. Finally, in Section $V I I$, the conclusions of the paper are provided.

\section{PRoBlem DEFINITION}

\section{A. Model Parametrisation}

Let us assume that the system can be expressed by a linear in parameters model that can be expressed in regressor form (MA model) as follows

$$
\begin{aligned}
& y(k)=\boldsymbol{\varphi}(k) \boldsymbol{\theta}(k)+e(k)=\hat{y}(k)+e(k) \\
& \boldsymbol{\theta}(k)=\boldsymbol{\theta}(k-1)+\boldsymbol{w}(k)
\end{aligned}
$$

where 
- $\boldsymbol{\varphi}(k)$ is the regressor vector of dimension $1 \times n_{\theta}$ which can contain any function of inputs $u(k)$ and outputs $y(k)$.

- $\boldsymbol{\theta}(k) \in \boldsymbol{\Theta}$ is the parameter vector of dimension $n_{\theta} \times 1$.

- $\Theta$ is the set that bounds parameter values.

- $e(k)$ is the additive error bounded by a constant $|e(k)| \leq \sigma$.

- $\boldsymbol{w}(k)$ is the parameter variation that it will be considered elementwise component bounded by a constant $\|\boldsymbol{w}(k)\|_{\infty} \leq \boldsymbol{\gamma}$.

In this paper, the bound parameter set $\boldsymbol{\Theta}$ is described by a polytope that can be expressed in the $\mathcal{H}$-polytope form [9] as

$$
\boldsymbol{\Theta}=\left\{\boldsymbol{\theta}(k) \in \mathbb{R}^{n_{\theta}} \mid \mathbf{A}_{0} \boldsymbol{\theta}(k) \leq \mathbf{b}_{0}\right\}
$$

with $\mathbf{A}_{0} \in \mathbb{R}^{n x n_{\theta}}$ and $\mathbf{b}_{0} \in \mathbb{R}^{n}$.

Notice that Eq. (2) specifies the allowed variance of uncertain parameters $\boldsymbol{\theta}$. Depending on the value of $\gamma$, in this paper two different cases will be considered.

- Time-invariant case, $\gamma=0$

- Bounded variance case, $\gamma \neq 0$

In the first case, the parameter is unknown within $\boldsymbol{\Theta}$, but it is known that it will not vary, whereas in the second case, the parameter variation is bounded by $\gamma$.

\section{B. Model Inconsistency}

Considering model (1) with variations in parameters (2) and bounding parameters set (3), an inconsistency (fault) is proved at instant $k$ when there not exist any parameter consistent with measurements and model conditions, and can be determined by checking if

$$
\begin{gathered}
\mathbf{A}_{\mathbf{0}} \boldsymbol{\theta}(i) \leq \mathbf{b}_{\mathbf{0}}, \forall i=1, \cdots, k \\
\neg \exists \boldsymbol{\theta}(i) \in \mathbb{R}^{n_{\theta}} I-\sigma \leq y(i)-\boldsymbol{\varphi}(i) \boldsymbol{\theta}(i) \leq \sigma, \forall i=1, \cdots, k \\
\|\boldsymbol{\theta}(i)-\boldsymbol{\theta}(i-1)\|_{\infty} \leq \gamma, \forall i=2, \cdots, k
\end{gathered}
$$

Fault detection test (4) can be performed by determining the feasibility of a linear problem of $k n+k+(k-1) n_{\theta}$ constrains and $k n_{\theta}$ variables with no objective function. Even being a linear problem, fault detection test (4) is very difficult to calculate when $k$ grows.

\section{SET-MEMBERShIP FAULT DETECTION}

\section{A. Set-membership Time-invariant Fault Detection}

Set-membership time-invariant fault detection considers no variation in parameters $\boldsymbol{\theta}(k)=\boldsymbol{\theta}(k-1)=\boldsymbol{\theta}$, i.e. $\boldsymbol{\gamma}=0$. And all the errors between the model and measurements are taken into account through the additive error bound $\sigma$.

At every instant $k$, a strip $\mathbf{F}_{k}$ can be obtained in the parameter space that contains all parameters consistent with measurement and regressor vector $\boldsymbol{\varphi}(k)$ defined by

$$
\mathbf{F}_{k}=\left\{\boldsymbol{\theta} \in \mathbb{R}^{n_{\theta}} \mid-\sigma \leq y(k)-\boldsymbol{\varphi}(k) \boldsymbol{\theta} \leq \sigma\right\}
$$

Then, the fault detection test consists in checking if the intersection of strip $\mathbf{F}_{k}$ with the Feasible Parameter Set, defined as the set that contains all parameters consistent with all previous data at instant $k$ leads to an empty set

$$
\mathbf{F}_{k} \cap \mathbf{F P S}_{k}=\varnothing
$$

The $\mathbf{F P S}_{\mathrm{k}}$ can be obtained by the intersection of strips from instant 1 to instant $k-1$ and the parameter set $\boldsymbol{\Theta}$

$$
\mathbf{F P S}_{k}=\left(\bigcap_{i=1}^{k-1} \mathbf{F}_{i}\right) \cap \boldsymbol{\Theta}
$$

Figure 1 shows an example, with $n_{\theta}=2$, of how to build the $\mathbf{F P S}_{\mathrm{k}}$ with one and three strips
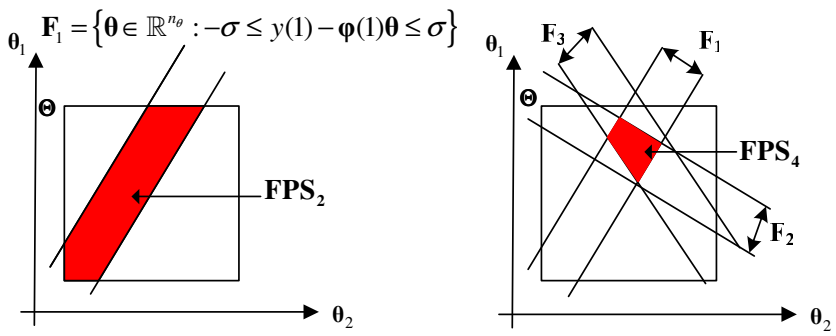

Figure 1. a) $\mathbf{F P S}_{2}$ : Intersection of strip $\mathbf{F}_{1}$ and the set $\boldsymbol{\Theta}$ b) $\mathbf{F P S}_{4}$ : Intersection of strips $\mathbf{F}_{1}, \mathbf{F}_{2}$ and $\mathbf{F}_{3}$ with the set $\boldsymbol{\Theta}$.

The feasible parameter set $\left(\mathbf{F P S}_{k}\right)$ is a polytope that can be described in the $\mathcal{H}$-polytope form as

$$
\mathbf{F P S}_{k}=\left\{\boldsymbol{\theta} \in \mathbb{R}^{n_{\theta}} \mid\left(\begin{array}{c}
\mathbf{A}_{0} \\
\mathbf{A}_{k-1}
\end{array}\right) \boldsymbol{\theta} \leq\left(\begin{array}{c}
\mathbf{b}_{0} \\
\mathbf{b}_{k-1}
\end{array}\right)\right\}
$$

with

$$
\mathbf{A}_{k-1} \in \mathbb{R}^{2(k-1) x n_{\theta}}=\left(\begin{array}{c}
-\varphi(1) \\
\varphi(1) \\
\vdots \\
-\varphi(k-1) \\
\varphi(k-1)
\end{array}\right), \mathbf{b}_{k-1} \in \mathbb{R}^{2(k-1)}=\left(\begin{array}{c}
-y(1)+\sigma \\
y(1)+\sigma \\
\vdots \\
-y(k-1)+\sigma \\
y(k-1)+\sigma
\end{array}\right)
$$

Then, fault test (6) boils down to determine the feasibility of the following linear problem

$$
\left(\begin{array}{l}
\mathbf{A}_{0} \\
\mathbf{A}_{k}
\end{array}\right) \boldsymbol{\theta} \leq\left(\begin{array}{l}
\mathbf{b}_{0} \\
\mathbf{b}_{k}
\end{array}\right)
$$

with $\mathbf{A}_{k}=\left(\begin{array}{c}\mathbf{A}_{k-1} \\ -\boldsymbol{\varphi}(k) \\ \boldsymbol{\varphi}(k)\end{array}\right) \quad$ and $\quad \mathbf{b}_{k}=\left(\begin{array}{c}\mathbf{b}_{k-1} \\ -y(k)+\sigma \\ y(k)+\sigma\end{array}\right)$

If linear problem (9) is no feasible then condition (6) is fulfilled and a fault is proved. On the other hand, if the linear problem (9) is feasible then the set of parameters consistent with data from instant 1 to instant $k\left(\mathbf{F P S}_{k+1}\right)$ is not empty and matrix $\left(\begin{array}{l}\mathbf{A}_{0} \\ \mathbf{A}_{k}\end{array}\right)$ and vector $\left(\begin{array}{l}\mathbf{b}_{0} \\ \mathbf{b}_{k}\end{array}\right)$ define the $\mathbf{F P S}_{k+1}$.

The main drawback of this detection test is that the number of constrains in the linear problem (9): $n+2 k$, grows with data. In order to avoid dealing with the exact description of the FPS, existing algorithms usually approximate the FPS using simpler shapes as boxes, parallelotopes, ellipsoids or 
zonotopes. The approximation set is called Approximated Feasible Parameter Set ( AFPS).

There are two kinds of approximations inner and outer approximations. Inner approximations guarantee that all the parameters of the approximate set are inside the feasible parameter set $\left(\mathbf{A F P S}_{k} \subseteq \mathbf{F P S}_{k}\right)$. Whereas outer approximations guarantee that the feasible parameter set is inside the approximation set ( $\mathbf{A F P S}_{k} \supseteq \mathbf{F P S}_{k}$ ). Since outer approximations include all the parameters consistent with data, they are preferable for fault detection purposes.

\section{B. Set-membership Time-varying Fault Detection}

In this section, the polytope-based set-membership fault detection approach presented in the previous section is extended to take into account the variation in model parameters (2). A similar approach can be found in [8] using zonotopes. Notice that in the proposed time-varying extension, the $\mathbf{F P S}_{k}$ (exact feasible parameter set) can not be computed anymore but instead an outer approximation denoted as $\mathbf{A F P S}_{k}$ will be used. The reason for this over approximation is due to all the possible parameter variations are taken into account by expanding the $\mathbf{F P S}_{k}$ to the

$\overline{\mathbf{A F P S}}_{k}$. The bounded varying case allows to take into account explicity parameter variations that with the invariant case are taken into account indirectly increasing the additive error bound $\sigma$.

The conceptual form of the suggested fault detection method is provided in Algorithm 1. The basic idea of the algorithm is as follows: At every time instant $k$, input/output system measurements obtained from sensors are used to build the regressor $\boldsymbol{\varphi}(k)$ and strip $\mathbf{F}_{k}$ according to (5). Then, the new set $\mathbf{A F P S}_{k+1}$ consistent with the strip and the polytope $\overline{\mathbf{A F P S}}_{k}$ is built. Then, the existence of this set is checked. In case an inconsistency is proved $\left(\mathbf{A F P S}_{k+1}=\varnothing\right)$, a fault is considered to be present. Otherwise, the algorithm proceeds to expand $\mathbf{A F P S}_{k+1}$ taking into account the allowed parameter variance $\gamma$.

Algorithm 1 Fault detection given $\gamma, \sigma$ and $\boldsymbol{\Theta}$

1: fault $\leftarrow$ FALSE
2: $k \leftarrow 1$
3: $\overline{\mathbf{A F P S}}_{k} \leftarrow \mathbf{\Theta}$
4: while fault $=$ FALSE do
5: $\quad$ Obtain input-output data $\{u(k), y(k)\}$ at time instant
$\quad k$ and build regressor $\varphi(k)$ and $\mathbf{F}_{k}$
6: $\quad$ AFPS $_{k+1}=\overline{\mathbf{A F P S}}_{k} \cap \mathbf{F}_{k}$
7: $\quad$ if AFPS $_{k+1}=\varnothing$
8: $\quad$ fault $=$ TRUE $_{\text {9: }} \quad$ else
10: $\quad$ Expand AFPS $_{k+1}$ taking into account $\gamma$ to obtain

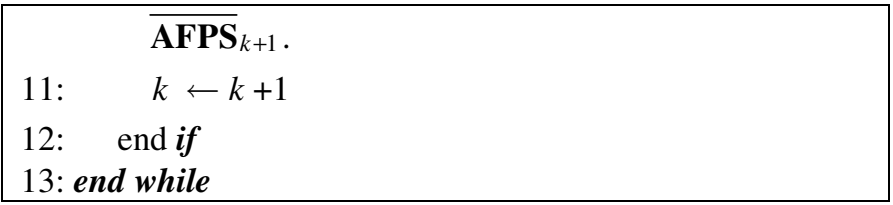

\section{IMPLEMENTATION OF THE FAULT DETECTION USING POLYTOPES}

\section{A. Intersection and Consistency}

The use of polytopes allows the computation of $\mathbf{A F P S}_{k+1}$ by adding two constrains associate to the strip $\mathbf{F}_{k}$ to the constraints of polytope $\overline{\mathbf{A F P S}}_{k}$.

On the other hand, checking if $\mathbf{A F P S}_{k+1}=\varnothing$ is equivalent to determine the feasibility of linear programming problem with no objective function and as constraints the polytope AFPS $_{k+1}$.

\section{B. Expansion of the parameter set}

Once the parameter set $\mathbf{A F P S}_{k+1}$ has been calculated, an extension of this parameter set has to be carried out in order to take into account parameter variation bound $\gamma$.

In order to compute this expansion, $\mathbf{F}_{i \mid k}(i \leq k)$ is defined as the strip of parameters at instant $k$ consistent with measuments at instant $i$, considering maximum effect of the variation between parameters (2) from time instant $i$ to $k$. i.e., $\mathbf{F}_{i \mid k}=\left\{\boldsymbol{\theta}(k) \in \mathbb{R}^{n_{\theta}} \mid-\left(\sigma+\Delta_{i \mid k}\right) \leq y(i)-\boldsymbol{\varphi}(i) \boldsymbol{\theta}(k) \leq \sigma+\Delta_{i \mid k}\right\}(11)$ where $\Delta_{i \mid k}$ is the deviation bound in the output estimation due to variation in parameters from time instant $i$ to $k \Delta \hat{y}_{i \mid k}$, that can be calculated as

$$
\Delta \hat{y}_{i \mid k}=\boldsymbol{\varphi}(i)(\boldsymbol{\theta}(k)-\boldsymbol{\theta}(i))=\sum_{j=i+1}^{k} \boldsymbol{\varphi}(i) \boldsymbol{w}(j)
$$

Then, considering parameter variation $\gamma$, (9) leads to

$$
\left|\Delta \hat{y}_{i \mid k}\right| \leq(k-i) \gamma\|\varphi(i)\|_{1}=\Delta_{i l k}
$$

Remark: Notice that $\mathbf{F}_{k \mid k}=\mathbf{F}_{k}$ from (5) and (11).

Then, the extension of AFPS $_{k+1}$ can be computed as

$$
\overline{\mathbf{A F P S}}_{k+1}=\left(\bigcap_{i=1}^{k} \mathbf{F}_{i \mid k}\right) \cap \boldsymbol{\Theta}
$$

Despite that the computation of the $\overline{\mathbf{A F P S}}_{k+1}$ with the strips $\mathbf{F}_{i \mid k}$ and the set $\boldsymbol{\Theta}$ is exact, the fact of the approximation is due to the use of the bound of $\Delta \hat{y}_{i \mid k}$ in (11) that guarantees the inclusion of all parameters consistent with data. This is why an outer approximation is obtained.

Figure 2 shows a graphical example of how to build $\mathbf{A F P S}_{k+1}$ and $\overline{\mathbf{A F P S}}_{k+1}$ with one and two measurements. 

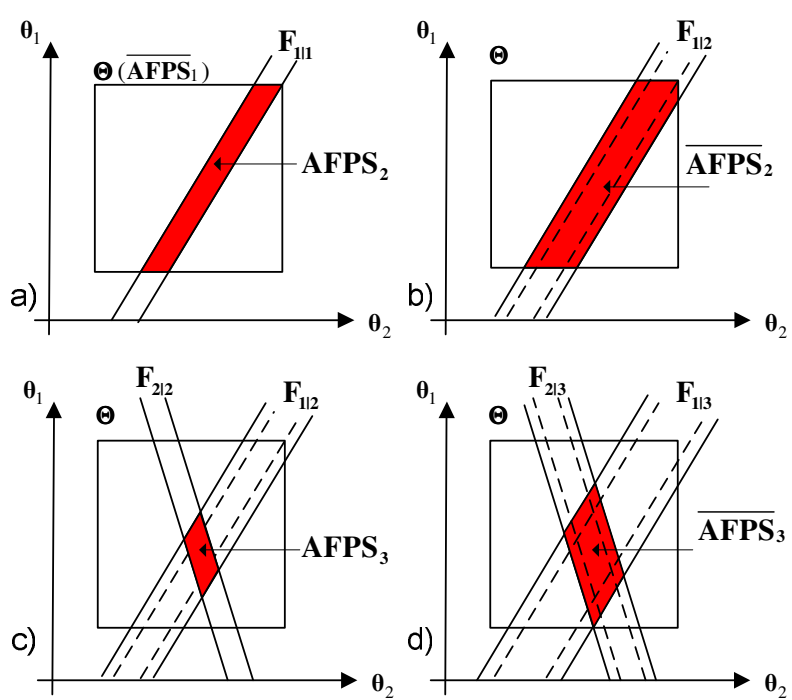

Figure 2. a) $\mathbf{A F P S}_{2}$ b) $\overline{\mathbf{A F P S}}_{2}$ c) $\mathbf{A F P S}_{3}$ d) $\overline{\mathbf{A F P S}}_{3}$

Considering the polytope $\mathbf{A F P S}_{k+1}$ is expressed as

$\mathbf{A F P S}_{k+1}=\left\{\boldsymbol{\theta}(k) \in \mathbb{R}^{n_{\theta}},\left(\begin{array}{l}\mathbf{A}_{0} \\ \mathbf{A}_{k}\end{array}\right) \boldsymbol{\theta}(k) \leq\left(\begin{array}{l}\mathbf{b}_{0} \\ \mathbf{b}_{k}\end{array}\right)+\gamma\left(\begin{array}{c}\mathbf{0} \\ \Delta \mathbf{b}_{k-1} \\ 0 \\ 0\end{array}\right)\right\}$

where $\Delta \mathbf{b}_{k-1} \in \mathbb{R}^{n_{A k}-2}$, with $n_{A k}$ the number of rows of $\mathbf{A}_{k}$, takes into account the previous expansions (from time instant 1 to time instant $k-1$ ). Then

$$
\begin{aligned}
& \overline{\mathbf{A F P S}}_{k+1}=\left\{\boldsymbol{\theta}(k+1) \in \mathbb{R}^{n_{\theta}},\left(\begin{array}{c}
\mathbf{A}_{0} \\
\mathbf{A}_{k}
\end{array}\right) \boldsymbol{\theta}(k+1) \leq\left(\begin{array}{c}
\mathbf{b}_{0} \\
\mathbf{b}_{k}
\end{array}\right)+\gamma\left(\begin{array}{c}
0 \\
\Delta \mathbf{b}_{k}
\end{array}\right)\right\}(16) \\
& \text { with } \Delta \mathbf{b}_{k}=\left(\begin{array}{c}
\Delta \mathbf{b}_{k-1 ; 1}+\left\|\mathbf{a}_{k ; 1,-}\right\|_{1} \\
\vdots \\
\Delta \mathbf{b}_{k-1 ; n_{A k}-2}+\left\|\mathbf{a}_{k ; n_{A k}-2},-\right\|_{1} \\
\left\|\mathbf{a}_{k ; n_{A k}-1,{ }_{-}}\right\|_{1} \\
\left\|\mathbf{a}_{k ; n_{A k}},-\right\|_{1}
\end{array}\right)
\end{aligned}
$$

where $\mathbf{a}_{k ; i},{ }_{-}$and $\Delta \mathrm{b}_{k-1 ; 1}$ are the $i^{\text {th }}$ row of the matrix $\mathbf{A}_{k}$ and the $i^{\text {th }}$ value of vector $\Delta \mathbf{b}_{k-1}$, respectively.

\section{Elimination of Redundant constrains}

As was showed in Section 3.A the main drawback of using polytopes in the feasible parameter set is that the number of constraints that define the polytope increases with $k$.

In the case of the proposed time-varying technique, the number of constraints of $\overline{\mathbf{A F P S}}_{k+1}$ is the same as in the invariant case: $n_{A k}=n+2 k$. In order to reduce the number of constraints once $\overline{\mathbf{A F P S}}_{k+1}$ has been calcultated, it has to be checked if there are constraints (except the constraints of the set $\boldsymbol{\Theta}$ ) that are redundant. If the constraint $i$ of the $\overline{\mathbf{A F P S}}_{k+1}$ is redundant [10], then the constraint (vector file $\mathbf{a}_{k ; i_{-}}$and values $\mathrm{b}_{k ; i}$ and $\Delta \mathrm{b}_{k ; i}$ ) can be removed from $\mathbf{A}_{k}$, $\mathbf{b}_{k}$ and $\Delta \mathbf{b}_{k}$.

The constraint elimination can also be carried out in the time-invariant fault detection procedure. But in the timevarying case, the fact that the strips $\mathbf{F}_{i k k}$ are expanded every instant $k$ guarantees that the constraints will be eliminated, in a time horizon that will depend on the value of $\gamma$, and the number of constraints will not grow indefinitely.

\section{Implementation of Algorithm 1 with Polytopes}

The procedure to implement Algorithm 1 in the case of using polytopes is described in Algorithm 2.

Algorithm 2 Implementation of Algorithm 1 with polytopes

$$
\text { 1: fault } \leftarrow \text { FALSE }
$$

2: $k \leftarrow 1$

3: $\overline{\mathbf{A F P S}}_{k} \leftarrow \boldsymbol{\Theta}\left(\mathbf{A}_{k-1}=0, \mathbf{b}_{k-1}=0\right.$ and $\left.\Delta \mathbf{b}_{k-1}=0\right)$

4: while fault $=$ FALSE do

5: $\quad$ Obtain input-output data $\{u(k), y(k)\}$ at time instant $k$ and build regressor $\boldsymbol{\varphi}(k)$

6: $\quad$ Build $\mathbf{A}_{k}$ and $\mathbf{b}_{k}$ as in (10)

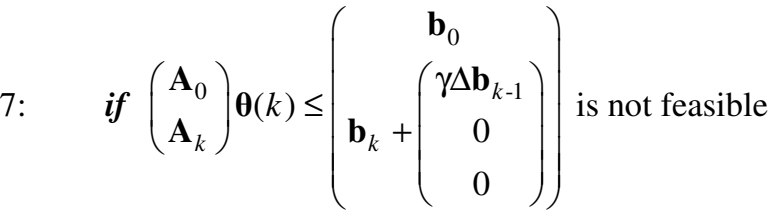

8: $\quad$ fault $=$ TRUE

9: $\quad$ else

10: $\quad$ Calculate $\Delta \mathbf{b}_{k}$ as in (17)

11: $\quad$ Reduce $\mathbf{A}_{k}, \mathbf{b}_{k}$ and $\Delta \mathbf{b}_{k}$

12: $\quad k \leftarrow k+1$

13: end if

14: end while

\section{PRACTICAL ISSUES}

Before applying the fault detection proposed in previous section the following issues should be determined: the structure of the model (determined by the components of the regressor $\boldsymbol{\varphi}(k))$, the set $\boldsymbol{\Theta}$ that bounds parameters and the constants $\sigma$ and $\gamma$ that determine the additive error of the model and the bound of the parameter variation.

The structure of the model can be determined by conventional identification methods. On the other hand, the parameter set $\boldsymbol{\Theta}$ can be obtained determining the physical possible bounds of parameters. Finally, the additive error $\sigma$ can be related to the sensor accuracy and noise.

Regarding the value of $\gamma$, the following procedure is proposed to determine its value: Given a sequence of data ( $M$ regressor vector values $\varphi(k)$ and measurement values $y(k))$ in a fault free scenario and rich enough from the identifiability point of view, the problem of finding the 
minimum variation bound in parameters $\gamma$ that guarantees consistency can be determined by solving Problem 1 Problem 1: "Set-membership parameter varying"

$\max \left\{\gamma_{k}\right\} \forall k=1, \ldots, M$

where $\gamma_{k}$ is calculated for every instant $k$ as min $\gamma$ subject to $\mathbf{A F P S}_{k+1} \neq \varnothing$

Problem 1 can be reformulated as $M$ optimization linear programing problems (one for every instant $k$ ) where a minimum variation bound in parameters $\gamma_{\kappa}$ is computed in order to guarantee $\mathbf{A F P S}_{k+1} \neq \varnothing$.

Problem 2: "Set-membership parameter varying (at particular $k$ )"

$\gamma_{k}=\left(n_{\theta}+1\right)$ component of $\arg \left(\min _{x} f x\right)$

subject to $\left(\begin{array}{cc}\mathbf{A}_{0} & \mathbf{0} \\ & -\Delta \mathbf{b}_{k-1} \\ \mathbf{A}_{k} & 0 \\ & 0\end{array}\right) \boldsymbol{x} \leq\left(\begin{array}{l}\mathbf{b}_{0} \\ \mathbf{b}_{k}\end{array}\right)$

with $\boldsymbol{f}=(0, \cdots, 0,1), \quad \boldsymbol{x}=\left(\boldsymbol{\theta}^{\mathrm{t}}(k) \quad \gamma\right)^{\mathrm{t}}$

Problem 1 can be solved by Algorithm 3 .

Algorithm 3 Estimation of $\gamma$ given $\sigma$ and $\boldsymbol{\Theta}$

1: Obtain a sequence of $M$ regressor vector values $\varphi(k)$ and measurement values $y(k)$

$2: k \leftarrow 1$

3: Obtain input-output data $\{u(k), y(k)\}$ at time instant $k$ and build regressor $\varphi(k)$

4: Build $\mathbf{A}_{k}=\left(\begin{array}{c}-\boldsymbol{\varphi}(k) \\ \boldsymbol{\varphi}(k)\end{array}\right), \mathbf{b}_{k}=\left(\begin{array}{c}-y(k)+\sigma \\ y(k)+\sigma\end{array}\right), \Delta \mathbf{b}_{k}=\left(\begin{array}{c}\|\boldsymbol{\varphi}(k)\|_{1} \\ \|\boldsymbol{\varphi}(k)\|_{1}\end{array}\right)$

5: $\gamma=0$

6: $k \leftarrow 2$

7: while $k \leq M$ do

8: Obtain input-output data $\{u(k), y(k)\}$ at time instant $k$ and build regressor $\boldsymbol{\varphi}(k)$

9: Build $\mathbf{A}_{k}$ and $\mathbf{b}_{k}$ as in (10).

10: Compute $\gamma_{k}$ solving Problem 2

11: $\gamma=\max \left(\gamma, \gamma_{k}\right)$

12: Compute $\Delta \mathbf{b}_{k}$ with (17)

13: Remove redundant constraints from $\mathbf{A}_{k}, \mathbf{b}_{k}, \Delta \mathbf{b}_{k}$

14: $k \leftarrow k+1$

15: end while

\section{APPLICATION}

\section{A. Application Description}

A quadruple-tank process, proposed by Johansson [11], will be used to illustrate the results presented in this paper. Schematic diagram of the system is shown in Figure 3a). The process inputs are the input voltages to the pumps ( $v_{1}$ and $\left.v_{2}\right)$ and the outputs are the tank levels $\left(h_{i}, i=1, \cdots, 4\right)$.
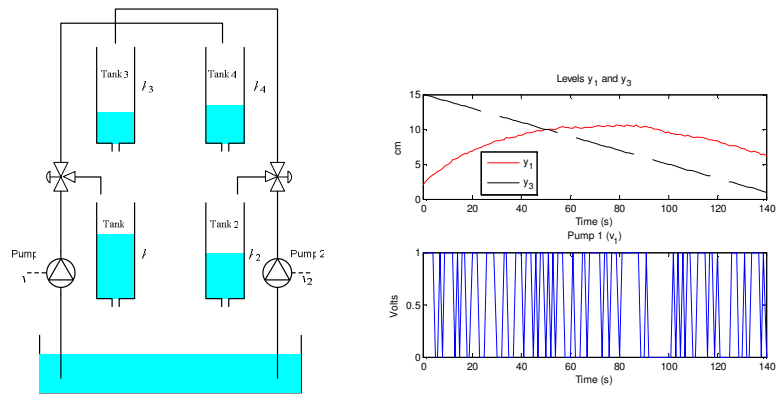

Figure 3: a) Quadruple-tank process.b) Fault free scenario

The experiments presented in this section just consider the residual coming from the first tank, assuming that levels $h_{1}$ and $h_{3}$ and voltage $v_{1}$ are measured

$$
\frac{d h_{1}}{d t}=-\frac{a_{1}}{A_{1}} \sqrt{2 g h_{1}}+\frac{a_{3}}{A_{1}} \sqrt{2 g h_{3}}+\frac{\rho_{1} k_{1}}{A_{1}} v_{1}
$$

where $a_{1}=a_{3}=0.071 \mathrm{~cm}^{2} A_{1}=28 \mathrm{~cm}^{2}, \quad k_{1}=3.33 \mathrm{~cm}^{3} / \mathrm{Vs}$, $g=981 \mathrm{~cm} / \mathrm{s}^{2}$ and $\rho_{1}=0.7$ assumed constants. It will be considered that the system is operating in the range

$$
h_{1} \in[2,11] \mathrm{cm} \text { and } h_{3} \in[1,15] \mathrm{cm}
$$

To obtain a model for fault detection in regressor form (1), Eq. (18) is discretised by the Euler method with sampling time $\Delta t=1 s$, then it can be expressed in regressor form (1) as follows

$y(k)=h_{1}(k), \varphi(k)=\left(h_{1}(k-1), h_{3}(k-1), \frac{k_{1} v_{1}(k-1)}{A_{1}}\right)$

$\boldsymbol{\theta}(k)=\left(a_{11}, a_{13}, \rho_{1}\right)^{t}$

with $a_{11}=1-\frac{a_{1}}{A_{1}} \sqrt{\frac{2 g}{h_{1}(k-1)}}$ and $a_{13}=\frac{a_{3}}{A_{1}} \sqrt{\frac{2 g}{h_{3}(k-1)}}$

And $e(k)$ is the additive error (including sensor and discretisation error) considered bounded $\left(\left|e_{1}(k)\right| \leq \sigma=0.05 \mathrm{~cm}\right)$.

\section{B. Alogrithm tuning}

In order to calibrate the model as was described in Section $V$, a fault free scenario with $M=140$ measurements has been recorded (Figure 3b).

The parameter set $\boldsymbol{\Theta}$ has been obtained by considering the operating range bounds (19) in (21) leading to the parameter bounds for $a_{11}$ and $a_{13}$

$$
a_{11} \in[0.92,0.97] \text { and } a_{13} \in[0.029,0.11]
$$

$\Theta$ can be expressed in the $\mathcal{H}$-polytope form (3) with

$$
\mathbf{A}_{0}=\left(\begin{array}{cc}
1 & 0 \\
-1 & 0 \\
0 & 1 \\
0 & -1
\end{array}\right) \quad \text { and } \quad \mathbf{b}_{0}=\left(\begin{array}{c}
0.97 \\
-0.92 \\
0.11 \\
-0.029
\end{array}\right)
$$

Then, with the model structure (20), the set $\boldsymbol{\Theta}$ defined by (23), the additive error bound $\sigma$ and the data of the fault 
free scenario, Algorithm 3 has been applied and minimum parameter variation bound $\gamma=0.001$ has been obtained.

\section{Fault Detection}

In order to show the behaviour of the fault detection method using polytopes and zonotopes, considering bounded variation in parameters, two different kind of faults have been considered: additive (in sensors) and a multiplicative (in components) faults. The fault scenarios are created introducing faults when the system is working in a fault free scenario.

Figures 4 and 5 show the behaviour of the fault detection test, implemented using Algorithm 2, in a non faulty scenario, with the parameters tuned in the previous section. Figure 4 shows the intersection of the initial set $\overline{\mathbf{A F P S}}_{1}=\boldsymbol{\Theta}$, with the first strip $\mathbf{F}_{1}$ and the new consistent sets $\mathbf{A F P S}_{2}$, calculated using the method proposed in Algorithm 2 and using the zonotope-based approach proposed in [8]. It can be noticed that the polytope-based approach provides the exact intersection, whereas the zonotope-based approach gives an outer approximation.
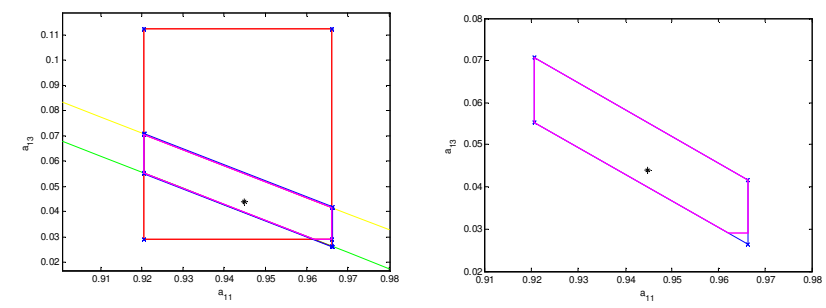

Figure 4: a) Intersection of the set $\overline{\mathbf{A F P S}}_{1}$ with the first strip $\mathbf{F}_{1}$, polytope (purple) and zonotope (blue) computations of $\mathbf{A F P S}_{2}$, and real vector parameter (21) (black point). b) Expansion of the calculated sets $\overline{\mathbf{A F P S}}_{2}$.

As can be noticed from Figure 4, the difference between the computations using a polytope or a zonotope are not significant in the first intersection. But this difference increases at every intersection (time step). For this reason polytope computation is more suitable for fault detection purposes. Figure 5 shows the sets $\overline{\mathbf{A F P S}}_{k+1}$ calculated at time instants $k=20$ and $k=40$.
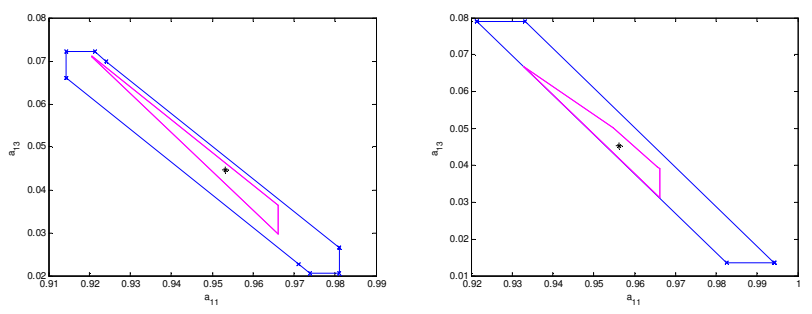

Figure 5: polytope and zonotope computations of $\overline{\mathbf{A F P S}} \quad$ a) at instant $k=20$ seconds b) at instant $k=40$ seconds

Figure 6 shows the result of the fault detection test in two simulated fault scenarios: a) "Output additive fault of 0.11 $\mathrm{cm}$ at $t=44 \mathrm{~s}$ " and b) "Multiplicative fault of $-0.012 \mathrm{~cm}^{2}$ in parameter $a_{l}$ of (18) at $t=44 \mathrm{~s}$.
In Figure 6 the polytope and zonotope based computations of $\overline{\mathbf{A F P S}}_{k}$ at $k=44$ and the strip at the time fault appearance are shown. As the strip does not intersect with the polytope the fault is detected in the polytope-based approach in both fault scenarios. On the other hand, as the strip intersects with the zonotope, the fault is not detected in the zonotope-based approach in neither of the two fault scenarios.
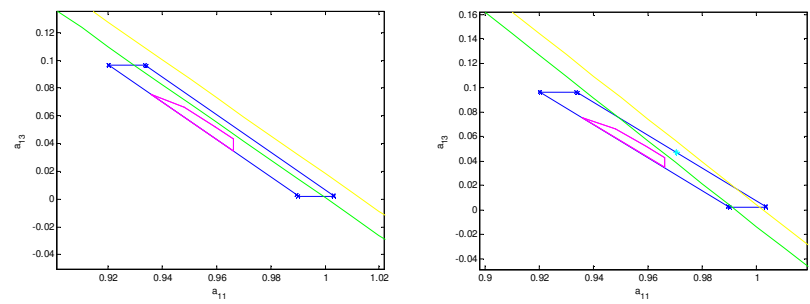

Figure 6: Polytope and zonotope computations of $\overline{\text { AFPS }}$ and strip of parameters consistent with measurements in fault scenario 1 a) and $2 \mathrm{~b}$ ), at the fault instant appearance $(\mathrm{t}=44 \mathrm{~s})$.

\section{CONCLUSIONS}

In this paper, a robust set-membership fault detection method that takes into account variation in model parameters is proposed. This methos is similar to the one proposed in [8] but uses polytopes instead of zonotopes to compute the set of parameters consistent with data. The conceptual description of the method, its implementation using polytopes and the algorithm tuning have been given. A case study based on a four-tank system has been used to show the effectiveness of the proposed fault detection method in different fault scenarios: additive and multiplicative faults.

\section{REFERENCES}

[1] J. Chen, R.J. Patton. (1999) Robust Model-Based Fault Diagnosis for Dynamic Systems, Kluwer Academic Publishers

[2] M. Milanese, J. Norton, H. Piet-Lahanier, E. Walter (eds). (1996) "Bounding Approaches to System Identification". Plenum Press.H.

[3] J. Watkins, S. Yurkovich Fault detection using set-membership identification. IFAC World Congress, San Francisco,U.S.A., 1996.

[4] A. Lesecq, K. Barraud, T. Dhin. Numerical accurate computations for ellipsoidal state bounding. Eleventh Mediterranean Conference on Control and Automation MED'03, Rhodes, Greece, 18-20 June 2003.

[5] A. Tzes, K. Le. Fault detection for jump discrete systems. IEEE Proceedings of the American Control Conference, San Diego, U.S.A., 1999.

[6] P. Kesavan, JH. Lee. A set based approach to detection and isolation of faults in multivariable systems. Computers and Chemical Engineering 2001; 25:925-940.

[7] P. Planchon, J. Lunze, (2008): Diagnosis of linear systems with structured uncertainties based on guaranteed state observation. International Journal of Control, Automation and Systems, 6, No. 3, pp. 308-319.

[8] A. Ingimundarson, V. Puig, T. Álamo, J.M Bravo and P. Guerra. (2008) Robust fault detection using zonotope-based set-membership consistency test. Journal of Adaptive Control and Signal Processing, 23(4): 311-330.

[9] G. M. Ziegler (1995). Lectures on Polytopes, Graduate Texts in Mathematics 152, Springer- Verlag New York Berlin Heidelberg.

[10] M H Karwan, V Lofti, J Telgen, S Zionts. Redundancy in Mathematical Programming: a State-of-the-Art Survey. Springer, Berlin, 1983.

[11] K.H. Johansson. (2000) "The quadruple-tank process: A multivariable laboratory with an adjustable zero". IEEE Transactions on Control Systems Technology, 8(3):456-465. 